Reprod. Nutr. Dévelop., 1988, 28 (6 B), 1747-1752

\title{
Stéroïdes sexuels et diagnostics de gestation chez les bovins - Tests en ferme
}

\author{
M. THIBIER
}

Union Nationale des Cooperatives d'Elevage et d'Insémination Artificielle, 13, rue Jouët. B.P. 65, 94703 Maisons-Alfort, France.

Summary. Steroids and pregnancy diagnosis in cattle - on the farm tests.

The peripheral blood and milk contain two steroids, whose concentrations can be monitored and used as a tool for pregnancy diagnosis in cattle: (1) Progesterone of maternal origin, which must be assayed between days 21-24 after Al, allows very early diagnosis of non pregnancy or of gestation onset, (2) Oestrone sulfate secreted by the feto-placental unit is a good means for confirming pregnancy over 110 days after Al.

Both steroids have been assayed in a very high number of samples by RIA in so-called centralized diagnosis laboratories. These have many advantages and usually are of a very high standard. Accuracy of these diagnoses has been evaluated in a large number of experimental series and is well known (93-100\% for early non pregnancy diagnosis).

ElA kits for progesterone have been on the market for $2-3$ years. But a more simple test to be used by the farmer himself is now available. It is a so-called dipstick test (Bovitest - ND - Clonatec), including control and test pads on the stick itself plus the conjugate and a soluble substrate. Reading and interpretation are made by single observation according to the intensity of the blue colour on the test pad compared to that on the control.

There is a good agreement between the results of the RIA procedure (used as a reference) and those of this dipstick test. A field study on a quite large basis was undertaken to evaluate the accurary of the diagnosis made according to this on the farm test. It was very similar to that found by RIA.

This kind of test used by the farmer, the Al technician or the clinician seems to be a most valuable tool for monitoring the productive status of an animal. It is yet to be determined what would be the expected pregnancy rate when an $\mathrm{Al}$ is performed the day a dipstick test is done on the farm and showed to be negative although no oestrus is recorded. This is being investigated at the present time. The preliminary results seem however quite encouraging.

Le diagnostic précoce et précis de la gestation chez les bovins est désormais un impératif économique. Associé à l'insémination artificielle, il permet une gestion efficace de la reproduction dans un troupeau de femelles reproductrices (Thibier, 1987).

II existe deux stéroïdes dont les concentrations dans le sang circulant ou le lait sont susceptibles d'apporter une telle information diagnostique: la progestérone et le sulfate d'œstrone (Laing et Heap, 1973; Thibier et al., 1973; Thibier, 1974; Heap et Hamon, 1979). Leur origine diffère, la première résulte de la sécrétion materne/le d'origine lutéale, nécessaire à l'initiation de la gestation. La 
mise à profit à des fins diagnostiques de cette activité endocrine repose donc sur la concentration différentielle de ce stéroïde à une période très précise, 21 à 24 jours après la fécondation espérée. Le diagnostic précoce est essentiellement recherché pour détecter dès que possible les femelles n'ayant pas initié de gestation, il correspond donc dans sa motivation à un diagnostic précoce de non-gestation.

Le sulfate d'œstrone est sécrété par l'unité fœto-placentaire elle-même et non par la mère, il est donc spécifique de la gestation. Il apparaît dans le sang circulant d'une façon distincte dès le début du second trimestre de cette gestation. II s'agit donc d'un diagnostic tardif et de confirmation de gestation, rendu parfois nécessaire quand les conditions d'élevage ne permettent pas une approche clinique ou échographique. Il est intéressant de noter que ces deux stéroïdes non seulement sont présents dans le plasma circulant mais aussi dans le lait. Leurs concentrations (de l'ordre $\mathrm{du} \mathrm{ng} / \mathrm{ml}$ ) sont donc dans ces deux tissus, d'excellents témoins de l'activité endocrine respective.

\section{Diagnostics radioimmunologiques en laboratoire.}

La radioimmunologie est la méthode de choix et de référence pour de tels dosages de stéroïdes (Thibier et al., 1973). Elle est employée en France depuis 15 ans environ et repose pratiquement sur un laboratoire centralisé, habilité et spécialisé, vers lequel convergent les échantillons. C'est le cas des deux laboratoires français principaux : celui de I'I.N.R.A. à Nouzilly pour les analyses liées aux protocoles de recherche et celui de I'UNCEIA à Maisons-Alfort concernant tous les prélèvements envoyés par les éleveurs dans le cadre de leur activité quotidienne. Au plan pratique, c'est un système qui fonctionne bien, le prélèvement est très simple dans le cas d'un échantillon de lait et l'analyse est peu coûteuse.

Les critères scientifiques d'exactitude (mesurant indirectement l'ampleur des erreurs par excès) et de fiabilité (mesurant indirectement ces erreurs par défaut) sont tout à fait satisfaisants ainsi que le rappelle le tableau 1, tiré des études de Thibier et Terqui (1978) et de Humblot et Thibier (1984).

Le facteur essentie/ de cette exactitude est la période de prélèvement qui doit être très scrupuleusement respectée : pour le diagnostic précoce de non-gestation tiré de l'analyse de la progestérone du plasma et du lait, cette période peut être seulement comprise entre $\mathrm{J} 21$ et $\mathrm{J} 24$ ( $\mathrm{JO}=$ jour de l'œestrus et donc de l'insémination) ainsi que l'illustre la figure 1. Pour le diagnostic de confirmation de la gestation par la mesure de l'œstrone sulfate, cette période ne peut être antérieure à 110 jours. Il faut remarquer que dans les conditions actuelles, l'exactitude obtenue pour ces deux types de tests précoces ou plus tardifs est identique quel que soit le mode de prélèvement: plasma ou lait.

La centralisation d'un tel laboratoire, rendue nécessaire par le recours aux radioisotopes entraîne naturellement un délai entre le moment du prélèvement de l'échantillon et le retour du résultat. Ce délai doit être le plus court possible. Dans notre service, tous les échantillons reçus un jour donné, sont analysés le jour 
TABLEAU 1

Taux d'exactitude des diagnostics de gestation par mesure de la concentration par RIA de la progestérone ou de l'œstrone sulfate dans le lait de vache aux jours appropriés $\left(^{*}\right)$.

\begin{tabular}{|c|c|c|c|c|c|c|c|}
\hline \multirow[b]{2}{*}{ Diagnostics } & \multirow[b]{2}{*}{ Stéroïdes } & \multirow{2}{*}{$\begin{array}{l}\text { Série } \\
\text { étudiée }\end{array}$} & \multirow[b]{2}{*}{ Nombre } & \multicolumn{2}{|c|}{ Exactitude (\%) } & \multirow{2}{*}{$\begin{array}{l}\text { Taux de } \\
\text { fertilité }\end{array}$} & \multirow[b]{2}{*}{ Référence } \\
\hline & & & & $\begin{array}{c}\text { Non } \\
\text { gestation }\end{array}$ & Gestation & & \\
\hline & & $\begin{array}{l}1 \\
2\end{array}$ & $\begin{array}{l}625 \\
113\end{array}$ & $\begin{array}{r}93 \% \\
100 \%\end{array}$ & $\begin{array}{l}62 \% \\
59 \%\end{array}$ & $\begin{array}{l}50 \% \\
28 \%\end{array}$ & (a) \\
\hline Précoce & Progestérone & $\begin{array}{l}3 \\
4 \\
5 \\
6\end{array}$ & $\begin{array}{l}466 \\
559 \\
502 \\
914\end{array}$ & $\begin{array}{l}94 \% \\
98 \% \\
99 \% \\
97 \%\end{array}$ & $\begin{array}{l}63 \% \\
79 \% \\
77 \% \\
68 \%\end{array}$ & $\begin{array}{l}54 \% \\
78 \% \\
60 \% \\
45 \%\end{array}$ & (b) \\
\hline Tardif & $\begin{array}{c}\text { Sulfate } \\
\text { d'œstrone }\end{array}$ & - & 147 & $100 \%$ & $97 \%$ & - & (b) \\
\hline
\end{tabular}

$\left({ }^{*}\right)$ Progestérone : J21-J24. Sulfate d'œstrone: au-delà de J110.

(a) Thibier et Terqui (1978); (b) Humblot et Thibier (1984).

même et les résultats renvoyés aussitôt. Plus de $80 \%$ des résultats sont connus de l'éleveur dans les $24 \mathrm{~h}$ suivant la réception des prélèvements au laboratoire. Ceci ne constitue aucun handicap pour le diagnostic de confirmation de gestation, le résultat n'est pas attendu à un jour près. Ceci est plus sensible pour le diagnostic précoce de gestation, et la connaissance du résultat d'un tel diagnostic sur place, au chevet de l'animal, peut présenter un intérêt clinique, thérapeutique et économique non négligeable pour le praticien et l'éleveur.

\section{Diagnostics par enzymo-immunologie sur bandelettes en ferme.}

L'essentiel de l'intérêt de connaître ce diagnostic sur le champ, résulte de la distorsion fréquente (voir Thibier et Terqui, 1978) entre la composante de comportement sexuel - l'œstrus - et la réalité de la phase folliculaire. Plus concrètement, un animal non vu en chaleur 21-23 jours après une insémination, donc pour lequel l'éleveur pense a priori qu'il est gestant peut en réalité ne pas l'être, se trouver en phase folliculaire et ovuler, à l'insu de celui-ci. La fréquence de tels animaux peut atteindre $20 \%$ de l'effectif inséminé (Humblot, 1986). Si le test est effectué sur place, l'éleveur peut tenter de faire inséminer sa vache immédiatement et ainsi espérer gagner plusieurs jours sur le moment de la fécondation par rapport à un résultat reçu tardivement, lorsque l'animal est déjà en phase lutéale et qu'il nécessite donc une thérapeutique lutéolytique. Le principe même de ce diagnostic précoce empêche de réaliser le test avant le $21^{\mathrm{e}}$ jour (voir fig. 1) donc de pouvoir bénéficier d'un délai de quelques jours entre le prélèvement et le moment optimal d'insémination dans l'éventualité d'une non-gestation. 


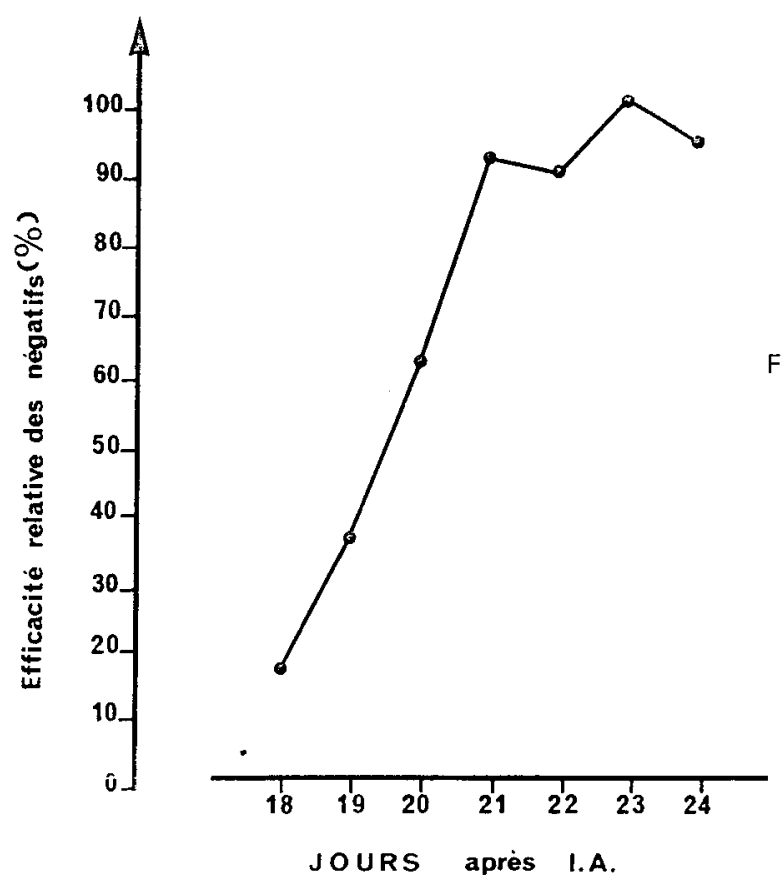

FIG. 1. - Efficacité relative d'un diagnostic précoce de gestation chez la vache selon le jour du prélèvement de lait après insémination (Humblot et al., 1987).

Ainsi plusieurs trousses de dosage, plutôt de type qualitatif, fondées sur l'ElA ont été mises sur le marché français, ces 3 dernières années. Leur manipulation est cependant difficilement comparable avec les habitudes gestuelles des éleveurs. Un des tests les plus intéressants à nos yeux, en raison même de son extrême simplicité est celui, dit sur bandelette (Bovitest - ND - Clonatec). L'éleveur peut facilement et rapidement $(9 \mathrm{~min})$ effectuer ce test. Ce test sur bandelette est pour le moment exclusivement adapté au lait, ce qui concorde logiquement avec l'objectif de simplicité recherché.

Le test met en œuvre :

$1^{\circ}$ Une bandelette dont l'extrémité comporte deux coussins : un témoin et un destiné au test. Sur celui-ci est fixé l'antigène - la progestérone.

$2^{\circ}$ Un anticorps monoclonal spécifique et conjugué à une enzyme, sous forme lyophilisée et qui sera mis en solution en présence du lait prélevé.

$3^{\circ}$ Un substrat soluble spécifique de l'enzyme conjuguée dans lequel sera trempée la bandelette après sa mise en présence de l'ensemble lait-conjugué (anticorps-enzyme).

La lecture et l'interprétation se fait par la simple observation de l'intensité de la couleur bleue du précipité par comparaison à celle du témoin.

Les premières analyses statistiques de concordance des résultats entre le RIA et le test sur bandelettes étant excellentes, nous avons mis en œuvre une étude à grande échelle de comparaison de l'exactitude de ces deux méthodes à partir de 
prélèvements effectués sur le terrain dans les conditions même de la pratique. Les résultats sont indiqués au tableau 2 , ils montrent à l'évidence une exactitude semblable tant pour les diagnostics de non-gestation que pour ceux de présomption de gestation.

TABLEAU 2

Exactitudes comparées du diagnostic de gestation par RIA ou ElA sur bandelettes ( ${ }^{*}$ ) (selon Humblot, comm. personnelle).

\begin{tabular}{l|c|c|c|c|c}
\hline \multirow{2}{*}{ Type d'élevage } & \multirow{2}{*}{ Méthode } & \multicolumn{2}{|c|}{ Exactitude } & \multirow{2}{*}{$\begin{array}{c}\text { Taux de } \\
\text { mise-bas }\end{array}$} & Nombre \\
\cline { 3 - 4 } & & Non-gestation & Gestation & \\
\hline 1 & RIA & $100 \%$ & $65,2 \%$ & $44,3 \%$ & 300 \\
(grands élevages) (a) & Bandelettes & $94 \%$ & $66,2 \%$ & & 123 \\
\hline 2 & RIA & $100 \%$ & $87,7 \%$ & & 324 \\
$\begin{array}{l}\text { (élevages de taille moyenne) } \\
\text { (b) }\end{array}$ & Bandelettes & $100 \%$ & $85,2 \%$ & $68,6 \%$ & 173 \\
\hline
\end{tabular}

$\left({ }^{*}\right)$ Bovitest (ND).

(a) Grands élevages : plus de 150 vaches en lactation.

(b) Elevages de taille moyenne : 30 à 60 vaches en lactation.

Suite à ces observations, des essais encore actuellement en cours, sont entrepris pour apprécier avec précision, les conséquences espérées sur la fertilité et le délai vêlage-conception, de I'Insémination Artificielle systématique sur femelles non vues en chaleur mais diagnostiquées avec certitude non gestantes 21 jours après l'insémination initiale. Les premiers résultats portant encore seulement sur une quinzaine d'animaux par lot (Humblot $e t$ al., com. personnelle) ne montrent pas d'effet défavorable significatif du recours à l'IA de façon systématique, indépendamment de l'absence de comportement sexuel observé.

En conclusion, les stéroïdes - la progestérone et le sulfate d'œstrone - sont deux témoins utilement mis à profit chez les bovins pour le diagnostic de présomption ou de confirmation de la gestation. Le lait, commode et simple à prélever est une excellente source biologique de cette information. L'éleveur dispose désormais pour le diagnostic précoce par la mesure de la concentration de progestérone, d'un choix entre un prélèvement très aisément effectué, expédié au laboratoire pour dosage RIA mais pour lequel il devra atteindre $48 \mathrm{~h}$ environ le résultat ou ce même prélèvement dont il effectuera l'analyse et qu'il interprétera lui-même au chevet de l'animal. Ce double choix permet plus de souplesse dans la gestion de la Reproduction des femelles, contribue à améliorer la technicité de l'atelier bovin et ainsi à augmenter l'efficacité économique du troupeau. 


\section{Références}

HEAP R. B., HAMON M., 1979. Oestrone sulfate in milk and its association with pregnancy. Br. Vet. J., 135, 462-463.

HUMBLOT P., 1986. La mortalité embryonnaire chez les bovins, 213-246. In Recherches récentes sur l'épidémiologie de la Fertilité. Coll. Soc. Fr. Fert. Masson (Paris).

HUMBLOT P., THIBIER M., 1984. Evaluation comparée des méthodes de diagnostic de gestation chez les bovins. El. Insém., N²00, 2-18.

HUMBLOT P., MAGNIN M., THIBIER M., SOMME G., 1987. Diagnostic précoce de non-gestation à la ferme chez les bovins. Choix du meilleur jour de prélèvement du lait. El. Insém., $\mathrm{N}^{\circ} \mathbf{2 1 9}$, $15-22$.

LAING J. A., HEAP R. B., 1973. The concentration of progesterone in the milk of cows during the reproductive cycle. Br. Vet. J., 127, 19-22.

THIBIER M., 1974. La progestérone dans le lait de vache. Diagnostic précoce de gestation. El. Insém., $\mathrm{N}^{\circ}$ 144, 27-32.

THIBIER M., 1987. L'insémination artificielle dans l'espèce bovine moyen privilégié d'améliorer l'efficacité de la reproduction. Ann. El. Bovins, 7-18, ITEB ed. (Paris).

THIBIER M., TEROUI M., 1978. Les diagnostics de gestation et de non-gestation chez les mammifères domestiques de ferme, 127-140. In Coll. Soc. Fr. Fert., Masson (Paris).

THIBIER M., CRAPLET C., PAREZ M., 1973. Les progestagènes naturels chez la vache. II. Conséquences zootechniques. Rec. Méd. vét., 149, 1601-1613. 